\title{
Formation Control of Multiple Dubin's Car System with Geometric Approach
}

\author{
Miswanto $^{1}$, I. Pranoto ${ }^{2}$, J. Naiborhu ${ }^{3}$, S. Achmadi ${ }^{4}$ \\ ${ }^{1}$ Department of Mathematics, Airlangga University, Indonesia \\ 2, 3, Industrial and Financial Mathematics Research Groups, ITB, Indonesia \\ ${ }^{4} \cdot$ Informatics Engeneering, ITN, Malang, Indonesia
}

\begin{abstract}
Formation control is an important behavior for multi-agents system (swarm). This paper addresses the optimal tracking control problem for swarm whose agents are Dubin's car moving together in a specific geometry formation. We study formation control of the swarm model which consists of three agents and one agent has a role as a leader. The agents of swarm are moving to follow the leader path. First, we design the control of the leader with tracking error dynamics. The control of the leader is designed for tracking the desired path. We show that the tracking error of the path of the leader tracing a desired path is sufficiently small. The desired path is obtained using calculus variational method. After that, geometry approach is used to design the control of the other. We show that the positioning and the orientation of each agent can be controlled dependent on the leader. The simulation results show to illustrate of this method at the last section of this paper.
\end{abstract}

Keywords - Swarm model, Dubin's car system, Tracking error, Calculus Variational, Numerical simulation.

\section{INTRODUCTION}

Natural phenomena are very interesting to analyze mathematically. One of the examples is the phenomenon called swarming which occurs in various groups of organisms. Swarming behavior or aggregation of organisms in groups is abundant in nature. For examples, the behavior can be seen in animal aggregation, flocks of birds (geese), schools of fish, herds of mammals etc. Flocks of geese often fly along in the inverted "V" formation. By flying in the inverted "V" formation they obtain some advantages. One of these advantages is that they are able to have $24 \%$ faster flying power and $71 \%$ greater flying range than if each goose flies on its own. One of the most interesting area is motion of swarm in [[10], [17]]. The motion of a swarm can be used in engineering for cooperative control (multi-robots) and formation control (aircraft and ship).

The formation preservation of the swarm have received considerable attentions. Gazi et al. [18], [19] and Miswanto, et. al. [15] study the coordination and tracking control problem of the motion of the swarm. In the literature, some researchers have discussed the formation control of mobile robots. In [14], the authors study a stable and decentralized control strategy for multi agent systems to capture a moving target in a specific formation. They use artificial potentials to take care both tracking and formation task. In [8], the authors study the stability properties of mobile agent formation which are based on leader following. They derive nonlinear gain estimates that capture how leader behavior affects the interconnection errors observed in the formation. In [16], the authors study a behavior based approach to robot formation preservation. In [13], the authors study control and coordination for many robots moving in formation using decentralized controllers. They investigate feedback law used to control multiple robots moving together in formation. They propose a method for controlling formations that uses only local sensor based information, in a leader-follower motion. They use methods of feedback linearization to exponentially stabilize the relative distance and orientation of follower and show that the zero dynamics of the system are also stable. In [11], the authors study the natural algebraic structure of the chained form system together with ideas from sliding mode theory while designing the control law. They consider sliding mode approach to the stabilization and tracking problfor the so called chained from nonholonomic system. In [6], the authors consider a problem of leader following in the case of a heterogeneous multi robot team. They use a discrete time sliding mode approach for control of nonholonomic robots performing a leader following task.

Another researchers have discussed the control design of a mobile robot to track a desired path. In [21], the authors discuss the tracking control of mobile robots using integrator backstepping. Many mechanical systems with nonholonomic contraints can be locally or globally converted to the chained form under coordinate change. In [22], the authors study the tracking control problem of nonholonomic system in chained form. They derive semi global tracking controllers for general chained form systems by means of backstepping and they achieve global tracking results for some special cases. In [4] an adaptive tracking control problem is studied for a four wheel mobile robot. The authors propose a formulation for the adaptive tracking problem that meets the natural prerequisite such that it reduces to the state feedback tracking problem if the parameters are known. They derive a general methodology for solving their problem. In [5], the authors study exponential tracking 
control of a mobile car using a cascaded approach. They show that the nonlinear controllers proposed in [21] can be simplified into linear controller for both the kinematic model and a simple dynamic model of the mobile robot. Their approach is based on cascaded system. In [2], the authors study a sufficient condition for the full state tracking stability of nonholonomic wheeled mobile robots by using the tracking control schemes based on the input output dynamics. They show that the tracking error internal dynamics and zero dynamics play a critical role of the full state tracking stability of such mobile robots. In [12], the authors propose a novel sliding mode control law for solving trajectory tracking problems of nonholonomic mobile robots. They use dynamic models of mobile robots to describe their behaviors with bounded disturbances in system dynamics. In [9], the authors propose a variable structure controller of a wheeled mobile robot to track the desired trajectory.

In this paper we consider the optimal tracking control problem for swarming behavior of three agents whose agents are moving to track a desired path in a desired geometric formation. We also compute the tracking error of the path of the leader tracing a desired path is sufficiently small and the distance between the leader path and the desired path is preserved. In the next section, the formal problem statement is described. In section 3 , we design the control of the leader using tracking error dynamics. In section 4, we design the control of each agent follower using geometric approach. In section 5 , we show numerical simulations to illustrate our results.

\section{Problem Statement}

Consider three Dubin's Car system described as:

$$
\begin{aligned}
\dot{x}_{i} & =u_{i} \cos \left(\theta_{i}\right) \\
\dot{y}_{i} & =u_{i} \sin \left(\theta_{i}\right) \\
\dot{\theta}_{i} & =\omega_{i} \\
Y_{i} & =\left[x_{i}, y_{i}, \theta_{i}\right]^{T}
\end{aligned}
$$

Where $\left(x_{i}, y_{i}\right) \in R^{2}$ denotes the position of the $i$-th Dubin's car, and $\theta_{i} \in[0,2 \pi]$ denotes the orientation of the car. The symbols $u_{i}$ and $\omega_{i}$ are the linear and angular velocities, respectively. Here, $Y_{i}$ is the output of the system. Thus, the output is the position and orientation of the car. In this paper, the desired path $\gamma$ that would be tracked by the leader is obtained using calculus variational method. The path is denoted by $\gamma(t)=\left(\gamma_{x}(t), \gamma_{y}(t)\right)$.

In this paper, there are two problems which will be discussed. First, we design the control of the leader. Furthermore, we design the control of the other agents by geometry approach to follow the leader's path with a certain distance.

\section{The Control Design Of The Leader}

We consider a model of the leader, such as (1). We design the control of the leader by tracking error dynamics for minimizing the tracking error in order to keep the position of the leader close to the desired path. We define a tracking error $e(t)$ as the difference between the actual leader path and the desired path:

$$
e(t)=\left[x_{1}(t)-\gamma_{x}(t), y_{1}(t)-\gamma_{y}(t)\right]^{\mathrm{T}}
$$

Differentiating the error equation (2) with respect to time yields.

$\dot{e}(t)=\left[\dot{x}_{1}(t)-\dot{\gamma}_{\mathrm{x}}(t), \dot{y}_{1}(t)-\dot{\gamma}_{\mathrm{y}}(t)\right]^{T}=\left[u_{1} \cos \left(\theta_{i}\right)-\dot{\gamma}_{\mathrm{x}}(t), u_{1} \sin \left(\theta_{i}\right)-\dot{\gamma}_{\mathrm{y}}(t)\right]^{T}$,

and

$$
\begin{aligned}
\ddot{e}(t) & =\left[\ddot{x}_{1}(t)-\ddot{\gamma}_{\mathrm{x}}(t), \ddot{y}_{1}(t)-\ddot{\gamma}_{\mathrm{y}}(t)\right]^{T} \\
& =\left[\dot{u}_{1} \cos \left(\theta_{i}\right)-u_{1} u_{2} \sin \left(\theta_{i}\right)-\ddot{\gamma}_{\mathrm{x}}(t), \dot{u}_{1} \sin \left(\theta_{i}\right)+u_{1} u_{2} \cos \left(\theta_{i}\right)-\ddot{\gamma}_{\mathrm{y}}(t)\right]^{T} .
\end{aligned}
$$

Now, we define the tracking error dynamics $F$ where $F=\left[f_{1}, f_{2}\right]^{T}$ and $f_{i}\left(e_{i}, \dot{e}_{i}\right)=0, i=1,2$

$$
\begin{aligned}
& f_{1}(t)=\dot{e}_{1}(t)+k_{1} e_{1}(t) \\
& f_{2}(t)=\dot{e}_{2}(t)+k_{2} e_{2}(t)^{\prime}
\end{aligned}
$$

where $k_{1}$ and $k_{2}$ are positive constants. Differentiating the system (5) with respect to time, one obtains

$$
\begin{aligned}
& \dot{f}_{1}(t)=\ddot{e}_{1}(t)+k_{1} \dot{e}_{1}(t) \\
& \dot{f}_{2}(t)=\ddot{e}_{2}(t)+k_{2} \dot{e}_{2}(t)^{\prime}
\end{aligned}
$$

The control of the leader can be determined directly from equations (1), (2), (3), (4), (5) and (6)

$w_{1}=\frac{\ddot{\gamma}_{y} \cos \left(\theta_{1}\right)+k_{2} \dot{\gamma}_{y} \cos \left(\theta_{1}\right)-\ddot{\gamma}_{x} \sin \left(\theta_{1}\right)-k_{1} \dot{\gamma}_{x} \sin \left(\theta_{1}\right)}{u_{1}}+\left(k_{1}-k_{2}\right) \sin \left(\theta_{1}\right) \cos \left(\theta_{1}\right)$,

where

$u_{1}=\dot{\gamma}_{x} \cos \left(\theta_{1}\right)+\dot{\gamma}_{y} \sin \left(\theta_{1}\right)+k_{1} \gamma_{\mathrm{x}} \cos \left(\theta_{1}\right)+k_{2} \gamma_{y} \sin \left(\theta_{1}\right)-k_{1} x_{1} \cos \left(\theta_{1}\right)-k_{2} y_{1} \sin \left(\theta_{1}\right)$.

Then, this control $\left(u_{1}, w_{1}\right)$ is substituted to the system (1). Thus, if one uses (7), one obtains a system of differential equations:

$$
\begin{aligned}
& \dot{x}_{1}=\left(\dot{\gamma}_{x} \cos \left(\theta_{1}\right)+\dot{\gamma}_{y} \sin \left(\theta_{1}\right)+k_{1} \gamma_{x} \cos \left(\theta_{1}\right)+k_{2} \gamma_{y} \sin \left(\theta_{1}\right)-k_{1} x_{1} \cos \left(\theta_{1}\right)-k_{2} y_{1} \sin \left(\theta_{1}\right)\right) \cos \left(\theta_{1}\right) \\
& \dot{y}_{1}=\left(\dot{\gamma}_{x} \cos \left(\theta_{1}\right)+\dot{\gamma}_{y} \sin \left(\theta_{1}\right)+k_{1} \gamma_{x} \cos \left(\theta_{1}\right)+k_{2} \gamma_{y} \sin \left(\theta_{1}\right)-k_{1} x_{1} \cos \left(\theta_{1}\right)-k_{2} y_{1} \sin \left(\theta_{1}\right)\right) \sin \left(\theta_{1}\right) \\
& \dot{\theta}_{1}=\frac{\ddot{\gamma}_{y} \cos \left(\theta_{1}\right)+k_{2} \dot{\gamma}_{y} \cos \left(\theta_{1}\right)-\dot{\gamma}_{x} \sin \left(\theta_{1}\right)-k_{1} \dot{\gamma}_{x} \sin \left(\theta_{1}\right)}{u_{1}}+\left(k_{1}-k_{2}\right) \sin \left(\theta_{1}\right) \cos \left(\theta_{1}\right) .
\end{aligned}
$$


The initial and final conditions of the state variables $\left(x_{1}, y_{1}, \theta_{1}\right)$ are known. The solution of this system of differential equations uses numerical approximation by substituting the desired path in system (8).

\section{The Control Design Of The Following Agents}

In this section, we design the control of the follower using geometry approach. Figure 1 shows three Dubin's cars. Where $d_{1}$ and $d_{2}$ are the distance of agents to the leader. $\varphi_{1}$ and $\varphi_{2}$ are the orientation of agents to position of the leader.

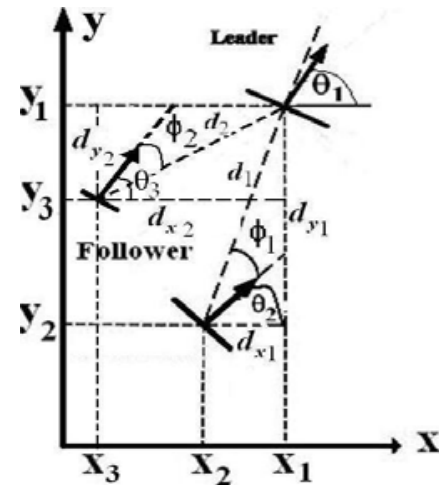

Figure 1: Three Dubin's cars

\section{IV.1 The Control Design of The First Follower}

From the figure 1 , we have

$x_{1}-x_{2}=d_{1} \cos \left(\theta_{2}+\varphi_{1}\right)$ and $y_{1}-y_{2}=d_{1} \sin \left(\theta_{2}+\varphi_{1}\right)$

Differentiating the equations above with respect to time, one obtains

$\dot{x}_{1}-\dot{x}_{2}=-d_{1} \sin \left(\theta_{2}+\varphi_{1}\right)\left(\dot{\theta}_{2}+\dot{\varphi}_{1}\right)$ and $\dot{y}_{1}-\dot{y}_{2}=d_{1} \cos \left(\theta_{2}+\varphi_{1}\right)\left(\dot{\theta}_{2}+\dot{\varphi}_{1}\right)$

Thus, we obtain

$\left(\dot{x}_{1}-\dot{x}_{2}\right) \cos \left(\theta_{2}+\varphi_{1}\right)+\left(\dot{y}_{1}-\dot{y}_{2}\right) \sin \left(\theta_{2}+\varphi_{1}\right)=0$

Substituting the system (1) for $i=1,2$ in the equation (10), one obtains

From the system (9), we have

$$
u_{2=} \frac{\dot{x}_{1} \cos \left(\theta_{2}+\varphi_{1}\right)+\dot{y}_{1} \sin \left(\theta_{2}+\varphi_{1}\right)}{\cos \left(\varphi_{1}\right)}
$$

$\left(\dot{x}_{1}-\dot{x}_{2}\right) \sin \left(\theta_{2}+\varphi_{1}\right)-\left(\dot{y}_{1}-\dot{y}_{2}\right) \cos \left(\theta_{2}+\varphi_{1}\right)=-d_{1}\left(\dot{\theta}_{2}+\dot{\varphi}_{1}\right)$

Therefore,

$$
\dot{x}_{1} \sin \left(\theta_{2}+\varphi_{1}\right)-\dot{y}_{1} \cos \left(\theta_{2}+\varphi_{1}\right)-u_{2} \sin \left(\varphi_{1}\right)+d_{1} \omega_{2}+d_{1} \dot{\varphi}_{1}=0
$$

From this equation, we obtain

$$
\omega_{2}=\frac{\dot{y}_{1} \cos \left(\theta_{2}+\varphi_{1}\right)-\dot{x}_{1} \sin \left(\theta_{2}+\varphi_{1}\right)-d_{1} \dot{\varphi}_{1}+\left(\dot{x}_{1} \cos \left(\theta_{2}+\varphi_{1}\right)+\dot{y}_{1} \sin \left(\theta_{2}+\varphi_{1}\right)\right) \tan \left(\varphi_{1}\right)}{d_{1}}
$$

Then, this control $\left(\omega_{2}, u_{2}\right)$ is substituted to the system (1) with $i=2$. Thus, we obtain a system of the differential equations of the first follower:

$$
\begin{aligned}
& \dot{x}_{2}=\frac{\dot{x}_{1} \cos \left(\theta_{2}+\varphi_{1}\right)+\dot{y}_{1} \sin \left(\theta_{2}+\varphi_{1}\right)}{\cos \left(\varphi_{1}\right)} \cos \left(\theta_{2}\right) \\
& \dot{y}_{2}=\frac{\dot{x}_{1} \cos \left(\theta_{2}+\varphi_{1}\right)+\dot{y}_{1} \sin \left(\theta_{2}+\varphi_{1}\right)}{\cos \left(\varphi_{1}\right)} \sin \left(\theta_{2}\right) \\
& \dot{\theta}_{2}=\frac{\dot{y}_{1} \cos \left(\theta_{2}+\varphi_{1}\right)-\dot{x}_{1} \sin \left(\theta_{2}+\varphi_{1}\right)-d_{1} \dot{\varphi}_{1}+\left(\dot{x}_{1} \cos \left(\theta_{2}+\varphi_{1}\right)+\dot{y}_{1} \sin \left(\theta_{2}+\varphi_{1}\right)\right) \tan \left(\varphi_{1}\right)}{d_{1}}
\end{aligned}
$$

\section{IV.2 The Control Design of The Second Follower}

From the figure 1 , we have $x_{1}-x_{3}=d_{2} \cos \left(\theta_{3}-\varphi_{2}\right)$ and $y_{1}-y_{3}=d_{2} \sin \left(\theta_{3}-\varphi_{2}\right)$

Using similar steps such as in 4.1., one may design the control of the second follower. Thus, we obtain a system of differential equations of the second follower: 
$\dot{x}_{3}=\frac{\dot{x}_{1} \cos \left(\theta_{3}-\varphi_{2}\right)+\dot{y}_{1} \sin \left(\theta_{3}-\varphi_{2}\right)}{\cos \left(\varphi_{2}\right)} \cos \left(\theta_{3}\right)$

$\dot{y}_{3}=\frac{\dot{x}_{1} \cos \left(\theta_{3}-\varphi_{2}\right)+\dot{y}_{1} \sin \left(\theta_{3}-\varphi_{2}\right)}{\cos \left(\varphi_{2}\right)} \sin \left(\theta_{3}\right)$

$\dot{\theta}_{3}=\frac{\dot{y}_{1} \cos \left(\theta_{3}-\varphi_{2}\right)-\dot{x}_{1} \sin \left(\theta_{3}-\varphi_{2}\right)-d_{2} \dot{\varphi}_{2}-\left(\dot{x}_{1} \cos \left(\theta_{3}-\varphi_{2}\right)+\dot{y}_{1} \sin \left(\theta_{3}-\varphi_{2}\right)\right) \tan \left(\varphi_{2}\right)}{d_{2}}$

\section{IV.2 Numerical Simulations}

In this section, some numerical simulations to illustrate the system (8), (11) and (12) are reported. For illustration, a desired path is the following parametric curve:

$$
\begin{aligned}
& \gamma_{1}(t)=\frac{11}{4000} t^{3}-\frac{33}{400} t^{2}+t+3 \\
& \gamma_{2}(t)=-\frac{11}{1000} t^{3}-\frac{3}{100} t^{2}+4
\end{aligned}
$$

The leader is expected to maneuver tracing this path as close as possible. The initial and final conditions of the leader are given by $\left(x_{1}(0), y_{1}(0), \theta_{1}(0)\right)=(3,4,0)$ and $\left(x_{1}(5), y_{1}(5), \theta_{1}(5)\right)=(12,8,0)$ respectively. The desired path in equation (17) is substituted in (10) and the parameters are $k_{1}=2$ and $k_{2}=2$. Fig. 2 shows the path of the leader tracing the desired path by using the method.

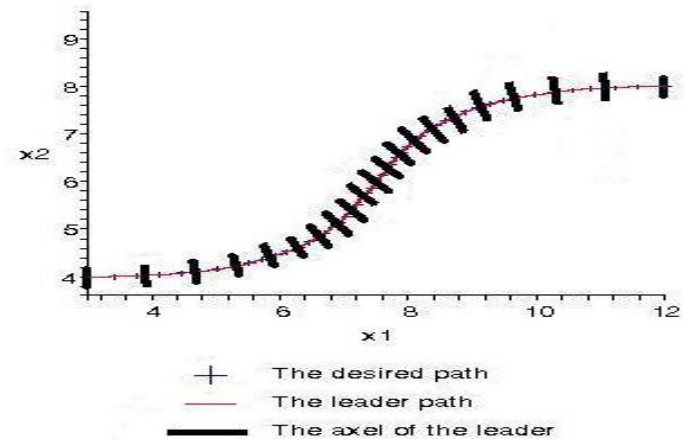

Figure 2: The trajectory of the desired path and the leader path.

Table 1. The tracking error

\begin{tabular}{|c|c|c|c|c|c|c|c|c|c|c|c|}
\hline Time $(\mathrm{t})$ & 0 & 1 & 2 & 3 & 4 & 5 & 6 & 7 & 8 & 9 & 10 \\
\hline Enror & 0 & 0 & 0 & $1 \mathrm{E}-08$ & $1 \mathrm{E}-08$ & $5 \mathrm{E}-08$ & $5 \mathrm{E}-08$ & $1 \mathrm{E}-07$ & $8 \mathrm{E}-08$ & $5 \mathrm{E}-08$ & $2 \mathrm{E}-08$ \\
\hline Time (t) & 11 & 12 & 13 & 14 & 15 & 16 & 17 & 18 & 19 & 20 \\
\hline Enror & $5 \mathrm{E}-08$ & $5 \mathrm{E}-08$ & $8 \mathrm{E}-08$ & $9 \mathrm{E}-08$ & $1.3 \mathrm{E}-07$ & 0 & $2 \mathrm{E}-08$ & $4 \mathrm{E}-08$ & $9 \mathrm{E}-08$ & $9 \mathrm{E}-08$ \\
\hline
\end{tabular}

Table 1 above shows the tracking error. Next, we show the numerical simulations to illustrate model (1) where $i=1,2,3$ in the two dimensional space. The movement of the two agents are described by the systems (13) and (16) and the leader is described by the system (10). The parame-ters $d_{1}, d_{2}=1$, $\varphi_{1}=\frac{1}{4} \pi$ and $\varphi_{2}=\frac{1}{18} \pi$. The initial values of the agents are given by $\left(x_{2}(0), y_{2}(0), \theta_{2}(0)\right)=$ $(2.13398,3.13398,-90)$ and $\left(x_{3}(0), y_{3}(0), \theta_{3}(0)\right)=(2.13398,4.86603,90)$. The results are presented in Fig. 3.

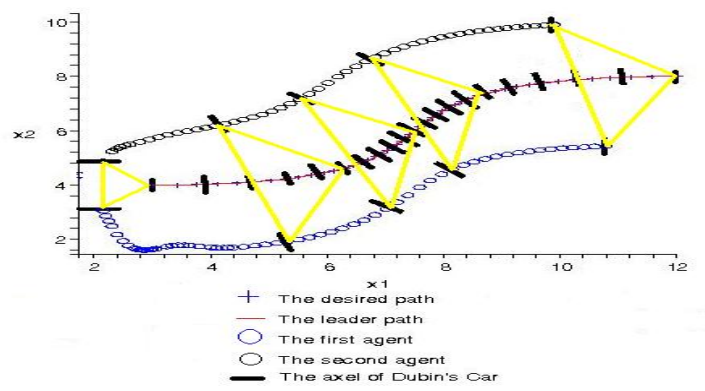

Figure 3: The trajectory of the swarm with three agent. 


\section{CONCLUSION}

A From the numerical simulation results above, it can be seen that the tracking error of the path of the leader tracing a desired path is sufficiently small and the distance between the leader path and the desired path is preserved. A geometry approach for formation control of a group of Dubin's car is investigated in this paper. The simulation on three Dubin's car formation demonstrates that the proposed method is effective and feasible. In future works, we will apply this method for movement control of another swarm model with a specific geometry formation.

\section{Acknowledgements}

Authors would like to thank the my institution (Airlangga University) for financial supporting this research. The authors would also like thanks Directorate General of Higher Education Department of National Education, Indonesia. This research was supported by Unggulan Perguruan Tinggi (UPT) grant under contract number 4074//H3.13/PPd/2012.

\section{REFERENCES}

[1] A. Balluchi, A. Bicchi, A. Balestrino, and G. Casalino, Path Tracking Control for Dubin's Cars, : Proceeding of the IEEE International Conference on Robotics and Automation, Volume: 4, pp. 3123-3128, 1998.

[2] D. Wang and G. Xu, Full State Tracking and Internal Dynamics of Nonholonomic Wheeled Mobile Robots, Proceedings of the American Control Conference, pp. 3274-3278, Chicago, Illinois, June 2000.

[3] E. Bicho and S. Monteiro, Formation Control for Multiple Mobile Robots: a Nonlinear Attractor Dynamics Approach, Proceeding of the IEEE/RSJ International Conference on Intelligent Robots and Systems (IROS 2003), Vol. 2, pp. 2016- $2022,2003$.

[4] E. Lefeber and H. Nijmeijer, Adaptive Tracking Control of Nonholonomic Systems: an example, Proceedings of the 38th Conference on Decision \& Control, Phoenix, Arizona USA, December 1999.

[5] E. Panteley, E. Lefeber, A. Loria and H. Nijmeijer, Exponential Tracking Control of a Mobile Car Using a Cascaded Approach, Proceeding of the IFAC Workshop on Motion Control, pp. 221-226, Grenoble France, September 1998.

[6] G. Orlando, E. Frontoni, A. Mancini, and P. Zingaretti, Sliding Mode Control for Vision Based Leader Following, 3rd European Conference on Mobile Robots, Freiburg, Germany, September 19-21 1999.

[7] G.Y. Tang, Y.D. Zhao and Hui Ma, Optimal Output Tracking Control for Bilinear Systems, Transactions of the Institute of Measurement and Control 28, 4, pp. 387-397, 2006.

[8] H.G. Tanner, G.J. Pappas, and V. Kumar, Leader to Formation Stability, IEEE Trans. on Robotics and Automation, Vol. 20 No. 3, June 2004.

[9] H.S. Shim, J. H. Kim, and K. Koh, Variable Structure Control of Nonholonomic Wheeled Mobile Robot, Proceeding of the IEEE International Conference on Robotics and Automation on Volume 2, Page(s):1694 - 1699, May 21-27 1995.

[10] H. Shi, L. Wang and T. Chu, Swarming Behavior of Multi-Agent Systems, J. Control Theory And Applications Vol. 2 No. 4, pp. 313-318, 2004.

[11] J. Lu, S. Sakhavat, M. Xie, and C. Lougier, Sliding Mode Control for Nonholonomic Mobile Robot, in : Proc. of the Int. Conf. on Control, Automation, Robotics and Vision, pp. 465-470, Singapore (SG), Dec. 2000.

[12] J.M. Yang and J.H. Kim, Sliding Mode Control for Trajectory Tracking of Nonholonomic Wheeled Mobile Robots, IEEE Trans. on Robotics and Automation, Vol. 15 No. 3, pp. 578-587, June 1999.

[13] J.P. Desai, J. Ostrowski, and V. Kumar, Controlling Formation of Multiple Mobile Robots, Proceeding of the IEEE International Conference on Robotics and Automation, Leuven, Belgium, May 1998.

[14] J. Yao, R. Ordonez, and V. Gazi, Swarm Tracking Using Artificial Potentials and Sliding Mode Control, Bio. Bull 202, pp. 296$305,2002$.

[15] Miswanto, I. Pranoto, and H. Muhammad, A Model of Swarm Movement with The Presence of A Leader, Proceeding of the International Conference on Mathematics and Natural Sciences, pp. 740-742, ITB, Bandung, 2006.

[16] T. Balch and R.C. Arkin, Behavior Based Formation Control for Multi-Robot Teams, IEEE Trans. on Robotics and Automation, Vol. XX No. y, Month 1999.

[17] V. Gazi and K.M. Passino, Stability Analysis of Swarms, IEEE Trans. on Automatic Control 48 No. 4, pp. 692-697, 2003.

[18] V. Gazi, B. Fidan, Y.S. Hanay, and M. Ilter Köksal, Aggregation, Foraging and Formation Control of Swarm with Non-Holonomic Agents Using Potential Functions and Sliding Mode Techniques, Turk. J. Elec. Engin. Vol. 15 No. 2, 2007.

[19] V. Gazi and R. Ordonez, Target Tracking Using Artificial Potentials and Sliding Mode Control, International Journal of Control, Vol. 80 No. 10, pp. 1626-1635, October 2007.

[20] Z. Jamaludin, H.V. Brussel, and J. Swevers, Classical Cascade and Sliding Mode Control Tracking performance for a XY Feed Table of a High-Speed Machine Tool, Int. J. Precision Technology, vol. 1, no. 1, 2007.

[21] Z-P. Jiang and H. Nijmeijer, Tracking Control of Mobile Robots:a Case Study in Backstepping, Automatica, vol. 33, no. 7, pp. 1393-1399, 1997.

[22] Z-P. Jiang and H. Nijmeijer, A Recursive Technique for Tracking Control of Nonholonomic Systems in Chained Form, IEEE Transaction on Automatic Control, vol. 44, no. 2, pp. 256-279, 1999b. 